\title{
Réponse à la lettre ouverte de la SSOT et de la SSU concernant les aide-mémoire pour les patients publiés par le Swiss Medical Board
}

Christoph Bosshard , Peter Suter ${ }^{b}$

a Comité central de la FMH, domaine DDQ

b Vice-président de l'Académie Suisse des Sciences Médicales (ASSM)
Les deux sociétés de discipline concernées donnent leur avis sur les travaux du Swiss Medical Board dans la lettre ouverte qui précède et à laquelle répondent les deux membres compétents de la FMH et de l'ASSM. Nous siégeons tous deux au Comité du Swiss Medical Board, une organisation cofinancée notamment par la FMH et l'ASSM et sommes convaincus que les points critiqués peuvent être améliorés grâce à une meilleure collaboration.
Le Swiss Medical Board est un organe indépendant de l'administration fédérale ou cantonale, des fournisseurs de prestations et de l'industrie. Sa tâche est d'analyser les processus diagnostiques et les interventions thérapeutiques du point de vue de la médecine, de l'économie, de l'éthique et du droit. L'objectif est de rédiger des rapports spécialisés afin de garantir des prestations médicales de qualité élevée et une utilisation efficace des ressources. Le Swiss Medical 
Board est géré conjointement par la CDS, l'ASSM, le gouvernement de la Principauté du Liechtenstein et la FMH. Il trouve son origine dans un projet de la Direction de la santé du canton de Zurich visant à mettre en œuvre les principes inscrits dans la LAMal concernant le HTA: l'art. 32 al. 1 LAMal exige en effet que les prestations mentionnées aux art. 25 à 31 doivent être efficaces, appropriées et économiques (critères «EAE») et que l'efficacité doit être démontrée selon des méthodes scientifiques. L'art. 32 al. 2 précise encore que les critères EAE doivent être réexaminés périodiquement.

Les sociétés de discipline sont associées aux travaux du Swiss Medical Board par l'intermédiaire d'un sondage annuel qui les invite à proposer les thèmes qu'elles entendent aborder. Ensuite, lors du traitement de ces thèmes, le Swiss Medical Board les consulte à nouveau pour qu'elles nomment des experts, et il part du principe que ces experts rédigent leurs prises de position en concertation avec les sociétés de discipline. Cependant, pour garantir la bonne collaboration de la FMH et de l'ASSM avec le Swiss Medical Board ainsi qu'une plus grande transparence à l'égard de notre base, il est important que les acteurs concernés - les sociétés de discipline médicale mais aussi les hôpitaux, les assureurs, les organisations de patients et les entreprises pharmaceutiques - soient à l'avenir également consultés avant la finalisation des recommandations définitives. Cela a été reçu positivement par le Comité du Swiss Medical Board lors de sa séance de novembre 2012. En effet, le Swiss Medical Board ne se concentrant pas uniquement sur des aspects purement médicaux, notamment en raison de ses objectifs, il est inévitable que des divergences de vue apparaissent avec les sociétés de discipline et les autres acteurs. Nous saisissons ici l'occasion pour rappeler l'éditorial du BMS n 48 [1] et l'histoire des six aveugles invités à décrire ensemble un éléphant. Comme eux, nous devons échanger nos points de vue en nous écoutant mutuellement mais aussi avoir la possibilité de prendre position.

La FMH et l'ASSM sont en train de discuter avec les autres membres du Swiss Medical Board dans le but de revoir les processus et de proposer des modifications allant dans le sens de ce qui est évoqué plus haut.

Pour de plus amples informations, veuillez consulter le site internet du Swiss Medical Board (www.swissmedicalboard.ch).

\section{Références}

1 Bosshard C. L'ASQM - le sixième sens du corps médical. Bull Méd Suisses. 2012;93(48):1775. 